Korean J. Math. 20 (2012), No. 2, pp. 239-245

\title{
APPLICATIONS OF LINKING INEQUALITIES TO AN ASYMMETRIC BEAM EQUATION
}

\author{
Q-Heung Choi and Tacksun JunG*
}

\begin{abstract}
We prove that an asymmetric beam equation has at least two solutions, one of which is a positive solution. To prove the existence of the other solution, we use linking inequalities.
\end{abstract}

\section{Introduction}

We investigate the existence of multiple solutions of the nonlinear beam equation in an interval $\left(-\frac{\pi}{2}, \frac{\pi}{2}\right)$,

$$
\begin{aligned}
& u_{t t}+u_{x x x x}+b u^{+}-\left|u^{-}\right|^{p-1}=f(x, t) \text { in }\left(-\frac{\pi}{2}, \frac{\pi}{2}\right) \times R, \\
& u\left( \pm \frac{\pi}{2}, t\right)=u_{x x}\left( \pm \frac{\pi}{2}, t\right)=0,
\end{aligned}
$$

$$
u \text { is } \pi \text {-periodic in } t \text { and even in } x \text { and } t \text {, }
$$

where the nonlinearity $-\left(b u^{+}\right)$crosses eigenvalues and $u^{+}=\max \{u, 0\}$, $u^{-}=\max \{-u, 0\}$. Here we suppose that $p>2$ and $f=s \phi_{00}+$ $\alpha h(x, t)(s>0), h$ is bounded. This equation represents a bending beam supported by cables under a load $f$. The nonlinearity $u^{+}$models the fact that cables resist expansion but do not resist compression.

Let $L$ be the differential operator, $L u=u_{t t}+u_{x x x x}$. Then the eigenvalue problem for $u(x, t)$

$$
L u=\lambda u \text { in }\left(-\frac{\pi}{2}, \frac{\pi}{2}\right) \times R
$$

with (2) and (3), has infinitely many eigenvalues

$$
\lambda_{m n}=(2 n+1)^{4}-4 m^{2} \quad(m, n=0,1,2, \cdots)
$$

Received April 29, 2012. Revised May 31, 2012. Accepted June 5, 2012.

2010 Mathematics Subject Classification: 35Q40, 35Q80.

Key words and phrases: critical point, linking inequality, multiple solutions.

This work was supported by Inha University Research Grant.

${ }^{*}$ Corresponding author. 
and corresponding eigenfunctions $\phi_{m n}(m, n \geq 0)$ given by

$$
\phi_{m n}=\cos 2 m t \cos (2 n+1) x
$$

We note that all eigenvalues in the interval $(-19,45)$ are given by

$$
\lambda_{20}=-15<\lambda_{10}=-3<\lambda_{00}=1<\lambda_{41}=17
$$
by

Let $\Omega$ be the square $\left[-\frac{\pi}{2}, \frac{\pi}{2}\right] \times\left[-\frac{\pi}{2}, \frac{\pi}{2}\right]$ and $H$ the Hilbert space defined

$$
H=\left\{u \in L^{2}(\Omega): u \text { is even in } x \text { and } t\right\} .
$$

Then the set of eigenfunctions $\left\{\phi_{m n}\right\}$ is an orthonormal base in $H$. Hence equation (1) with (2) and (3) is equivalent to

$$
L u+b u^{+}=f \text { in } H .
$$

In [6], the authors showed by degree theory that equation (4) with constant load $1+\epsilon h$ ( $h$ is bounded ) has at least two solutions. In [1], the authors showed by a variational reduction method that equation (4) with constant load $1+\epsilon h$ ( $h$ is bounded ) has at least three solutions when condition (3) is replaced by

$$
u \text { is } \pi \text {-periodic in } t \text { and even in } x \text {. }
$$

In [5], the author showed by linking method and category theory that the following asymmetric beam equation has multiple nontrivial solutions

$$
L u+b u^{+}=\left|u^{+}\right|^{p-1}-\left|u^{-}\right|^{q-1} \text { in } H .
$$

McKenna and Walter [7] proved that if $3<b<15$ then at least two $\pi$ periodic solutions exist, one of which is large in amplitude. The existence of at least three solutions was later proved by Choi, Jung and McKenna [2] using a variational reduction method. Humphreys [4] proved that there exists an $\varepsilon>0$ such that when $15<b<15+\varepsilon$ at least four periodic solutions exist. Choi and Jung [1] suppose that $3<b<15$ and $f$ is generated by eigenfunctions. Since Micheletti and Saccon [8] applied the limit relative category to studying multiple nontrivial solutions for a floating beam.

The main result of this paper is the following.

Theorem 1.1. Let $\Lambda_{i}^{-}<-b(b>0)$ and $f=s e_{1}^{+}(s>0)$. Let $u_{p}$ be the positive solution of (1). Then problem (1) has at least two solutions, one of which is a positive solution. 
In this paper, we use a variational approach and look for critical points of a suitable functional $I$ on a Hilbert space $H$. In Section 2, we find a suitable functional $I$ on a Hilbert space $H$ and prove the suitable version of the Palais-Smale condition for the topological method. In Section 3, we study the geometry of the sub-levels of $I$ and find two linking type inequalities, relative to two different decompositions of the space $H$.

\section{The Palais Smale condition}

To begin with, we consider the associated eigenvalue problem

$$
\begin{gathered}
L u=\lambda u \quad \text { in }\left(-\frac{\pi}{2}, \frac{\pi}{2}\right) \times R \\
u\left( \pm \frac{\pi}{2}, t\right)=u_{x x}\left( \pm \frac{\pi}{2}, t\right)=0 \\
u(x, t)=u(-x, t)=u(x,-t)=u(x, t+\pi) .
\end{gathered}
$$

A simple computation shows that equation (3) has infinitely many eigenvalues $\lambda_{m n}$ and the corresponding eigenfunctions $\phi_{m n}$ given by

$$
\begin{gathered}
\lambda_{m n}=(2 n+1)^{4}-4 m^{2}, \\
\phi_{m n}(x, t)=\cos 2 m t \cos (2 n+1) x \quad(m, n=0,1,2, \cdots) .
\end{gathered}
$$

Let $\Omega$ be the square $\left[-\frac{\pi}{2}, \frac{\pi}{2}\right] \times\left[-\frac{\pi}{2}, \frac{\pi}{2}\right]$ and $H$ the Hilbert space defined by

$$
H=\left\{u \in L^{2}(\Omega) \mid u \text { is even in } x \text { and } t\right\} .
$$

Then the set $\left\{\phi_{m n} \mid m, n=0,1,2, \cdots\right\}$ is an orthogonal base of $H$ and $H$ consists of the functions

$$
u(x, t)=\sum_{m, n=0}^{\infty} a_{m n} \phi_{m n}(x, t)
$$

with the norm given by

$$
\|u\|^{2}=\sum_{m, n=0}^{\infty} a_{m n}^{2} .
$$

We denote by $\left(\Lambda_{i}^{-}\right)_{i \geq 1}$ the sequence of the negative eigenvalues of equation (3), by $\left(\Lambda_{i}^{+}\right)_{i \geq 1}$ the sequence of the positive ones, so that

$$
\cdots<\Lambda_{1}^{-}=-3<\Lambda_{1}^{+}=1<\Lambda_{2}^{+}=17<\cdots .
$$

We consider an orthonormal system of eigenfunctions $\left\{e_{i}^{-}, e_{i}^{+}, i \geq 1\right\}$ associated with the eigenvalues $\left\{\Lambda_{i}^{-}, \Lambda_{i}^{+}, i \geq 1\right\}$. 
The following theorem is the uniqueness result.

Proposition 2.1. Let $b<-\Lambda_{1}^{-}$and $p>2$. Then the equation

$$
L u+b u^{+}-\left|u^{-}\right|^{p-1}=0 \text { in } H
$$

has only the trivial solution.

Proof. We rewrite the above equation as

$$
\begin{aligned}
L u-\Lambda_{1}^{+} u & =-\Lambda_{1}^{+} u-b u^{+}+\left|u^{-}\right|^{p-1} \\
& =-\Lambda_{1}^{+} u^{+}-b u^{+}+\left|u^{-}\right|^{p-1}+\Lambda_{1} u^{-}+\left|u^{-}\right|^{p-1} .
\end{aligned}
$$

Multiplying across by $e_{1}^{+}$and integrating over $\Omega$,

$$
\begin{aligned}
0 & =<\left[L-\Lambda_{1}^{+}\right] u, e_{1}^{+}> \\
& =\int_{\Omega}\left(-\Lambda_{1}^{+} u^{+}-b u^{+}+\left|u^{-}\right|^{p-1}+\Lambda_{1} u^{-}+\left|u^{-}\right|^{p-1}\right) e_{1}^{+} d x d t \geq 0,
\end{aligned}
$$

since the condition $b<-\Lambda_{1}^{-}$imply that $-\Lambda_{1}^{+} u^{+}-b u^{+}+\left|u^{-}\right|^{p-1}+\Lambda_{1} u^{-}+$ $\left|u^{-}\right|^{p-1} \geq 0$ for all real valued functions $u$ and $e_{1}^{+}(x)>0$ for all $x \in \Omega$. Therefore the only possibility to hold (1) is that $u \equiv 0$.

Theorem 2.2. Let $b<-\Lambda_{1}^{-}, s>0$ and $\|h\|=1$. Then there exists $\alpha_{0}>0$ such that for $\alpha<\alpha_{0}$ the equation

$$
L u+b u^{+}+\left|u^{-}\right|^{p-1}=s e_{1}^{+}+\alpha h(x, t) \text { in } H
$$

has a positive solution.

Proof. Since $b<-\Lambda_{1}^{-}<-\Lambda_{1}^{+}, b+\Lambda_{1}^{+}>0$ Thus the equation

$$
L u+b u^{+}=s e_{1}^{+} \text {in } H
$$

has a positive solution $u_{p}=\frac{s}{b+\Lambda_{1}^{+}} e_{1}^{+}$, which is a positive solution of the equation

$$
L u+b u^{+}+\left|u^{-}\right|^{p-1}=s e_{1}^{+} \text {in } H .
$$

Therefore there exists $\alpha_{0}>0$ such that for $\alpha<\alpha_{0}$ equation (1) has a positive solution.

We set

$$
\begin{aligned}
& H^{+}=\text {closure of } \operatorname{span}\{\text { eigenfunctions with eigenvalue } \geq 0\}, \\
& H^{-}=\text {closure of } \operatorname{span}\{\text { eigenfunctions with eigenvalue } \leq 0\} .
\end{aligned}
$$

We define the linear projections $P^{-}: H \rightarrow H^{-}, P^{+}: H \rightarrow H^{+}$. 
We also introduce two linear operators $R: H \rightarrow H^{+}, S: H \rightarrow H^{-}$by

$$
S(u)=\sum_{i=1}^{\infty} \frac{a_{i}^{-} e_{i}^{-}}{\sqrt{-\Lambda_{i}^{-}}}, R(u)=\sum_{i=1}^{\infty} \frac{a_{i}^{+} e_{i}^{+}}{\sqrt{\Lambda_{i}^{+}}}
$$

if

$$
u=\sum_{i=1}^{\infty} a_{i}^{-} e_{i}^{-}+\sum_{i=1}^{\infty} a_{i}^{+} e_{i}^{+} .
$$

It is clear that $S$ and $R$ are compact and self adjoint on $H$.

Definition 2.3. Let $I_{b}: H \rightarrow R$ be defined by

$$
I_{b}(u)=\frac{1}{2}\left\|P^{+} u\right\|^{2}-\frac{1}{2}\left\|P^{-} u\right\|^{2}+\frac{b}{2}\left\|[A u]^{+}\right\|^{2}-\int_{\Omega} G(A u) d x d t
$$

where $A=R+S$ and $G(s)=\int_{0}^{s} g(x, t, \tau) d \tau, g(x, t, \tau)=s e_{1}^{+}-\left|\tau^{-}\right|^{p-1}$.

It is straightforward that

$$
\nabla I_{b}(u)=P^{+} u-P^{-} u+b A(A u)^{+}-A g(A u) .
$$

Following the idea of Hofer (see [3]) one can show that

Proposition 2.4. $I_{b} \in C^{1,1}(H, R)$. Moreover $\nabla I_{b}(u)=0$ if and only if $w=(R+S)(u)$ is a weak solution of $(P)$, that is,

$$
\int_{\Omega}\left(w\left(v_{t t}+v_{x x x x}\right)+b[w]^{+} v\right) d x d t=\int_{\Omega} g(w) v d x d t
$$

for all smooth $v \in H$.

In this section, we suppose $b>0$. Under this assumption, we have a concern with multiplicity of solutions of equation (1). Here we suppose that $f$ is defined by equation (2).

In the following, we consider the following sequence of subspaces of $L^{2}\left(R^{N}\right)$ :

$$
H_{n}=\left(\oplus_{i=1}^{n} H_{\Lambda_{i}^{-}}\right) \oplus\left(\oplus_{i=1}^{n} H_{\Lambda_{i}^{+}}\right)
$$

where $H_{\Lambda}$ is the eigenspace associated to $\Lambda$.

Lemma 2.5. The functional $I_{b}$ satisfies $(P . S .)_{\gamma}^{*}$ condition, with respect to $\left(H_{n}\right)$, for all $\gamma$.

For the proof we refer [2], [5]. 


\section{Linking theory and main result}

Fixed $\Lambda_{i}^{-}$and $\Lambda_{i}^{-}<-b<\Lambda_{i-1}^{-}$. We prove the Theorem via a linking argument.

First of all, we introduce a suitable splitting of the space $H$. Let

$$
Z_{1}=\oplus_{j=i+1}^{\infty} H_{\Lambda_{j}^{-}}, Z_{2}=H_{\Lambda_{i}^{-}}, Z_{3}=\oplus_{j=1}^{i-1} H_{\Lambda_{j}^{-}} \oplus H^{+}
$$

Lemma 3.1. There exists $R$ such that $\sup _{v \in Z_{1} \oplus Z_{2},\|v\|=R} I_{b}(v) \leq 0$.

Proof. If $v \in Z_{1} \oplus Z_{2}$ then

$$
I_{b}(v)=-\frac{1}{2}\|v\|^{2}+\frac{b}{2}\left\|[S v]^{+}\right\|^{2}-\int_{\Omega} G(S v) d x d t .
$$

Since

$$
\frac{b}{2}\left\|[S v]^{+}\right\|^{2}-\int_{\Omega} G(S v) d x=\int_{\Omega} \frac{b}{2}\left([S v]^{+}\right)^{2}-\frac{1}{p}\left([S v]^{-}\right)^{p} d x d t
$$

there exists $R$ such that $-\frac{1}{4}\|v\|^{2}+\frac{b}{2}\left\|[S v]^{+}\right\|^{2}-\int_{\Omega} G(S v) d x \leq 0$ for all $\|v\|=R$. Hence

$$
I_{b}(v) \leq-\frac{1}{4}\|v\|^{2} \leq 0
$$

Lemma 3.2. There exists $\rho$ such that $\inf _{u \in Z_{2} \oplus Z_{3},\|u\|=\rho} I_{b}(u)>0$.

For the proof we refer [5].

DeFinition 3.3. Let $H$ be an Hilbert space, $Y \subset H, \rho>0$ and $e \in H \backslash Y, e \neq 0$. Set:

$$
\begin{aligned}
B_{\rho}(Y) & =\{x \in Y \mid\|x\| \leq \rho\}, \\
S_{\rho}(Y) & =\{x \in Y \mid\|x\|=\rho\}, \\
\triangle_{\rho}(e, Y) & =\{\sigma e+v \mid \sigma \geq 0, v \in Y,\|\sigma e+v\| \leq \rho\}, \\
\Sigma_{\rho}(e, Y) & =\{\sigma e+v \mid \sigma \geq 0, v \in Y,\|\sigma e+v\|=\rho\} \cup\{v \mid v \in Y,\|v\| \leq \rho\} .
\end{aligned}
$$

Theorem 3.4. Let $\Lambda_{i}^{-}<-b(b>0)$ and $f=s e_{1}^{+}(s>0)$. Let $u_{p}$ be the positive solution of (1). Then problem (1) has at least two solutions, one of which is a positive solution. 
Proof. Let $e \in Z_{2}$. By Lemma 3.1 and Lemma 3.2, for a suitable large $R$ and a suitable small $\rho$, we have the linking inequality

$$
\sup I_{b}\left(\Sigma_{R}\left(e, Z_{1}\right)\right)<\inf I_{b}\left(S_{\rho}\left(Z_{2} \oplus Z_{3}\right)\right) \text {. }
$$

Moreover (P.S. $)_{\gamma}^{*}$ holds. By standard linking arguments, it follows that there exists a critical point $u$ for $I_{b}$ with $\alpha \leq I_{b}(u) \leq \beta$, where $\alpha=$ $\inf I_{b}\left(S_{\rho}\left(Z_{2} \oplus Z_{3}\right)\right)$ and $\beta=\sup I_{b}\left(\Delta_{R}\left(e, Z_{1}\right)\right)$. Since $\alpha>0$ and $I_{b}\left(u_{p}\right)=$ $0, u \neq u_{p}$. Therefore then problem (1) has at least two solutions, one of which is a positive solution.

\section{References}

[1] Q.H. Choi, T.S Jung, A nonlinear suspension bridge equation with nonconstant load, Nonlinear Anal. 35 (1999), 649-668.

[2] Q.H. Choi, T. Jung, P.J. McKenna, The study of a nonlinear suspension bridge equation by a variational reduction method, Appl. Anal. 50 (1993), 71-90.

[3] H. Hofer, On strongly indefinite functionals with applications, Trans. Amer. Math. Soc. 275 (1983), 185-214.

[4] L. Humphreys, Numerical and theoretical results on large amplitude periodic solutions of a suspension bridge equation, ph.D. thesis, University of Connecticut (1994).

[5] S. Li, A. Squlkin, Periodic solutions of an asymptotically linear wave equation, Nonlinear Anal. 1 (1993), 211-230.

[6] J.Q. Liu, Free vibrations for an asymmetric beam equation, Nonlinear Anal. 51 (2002), 487-497.

[7] P.J. McKenna, W. Walter, Nonlinear Oscillations in a Suspension Bridge, Arch. Ration. Mech. Anal. 98 (1987), 167-177.

[8] A.M. Micheletti, C. Saccon, Multiple nontrivial solutions for a floating beam via critical point theory, J. Differential Equations, 170 (2001), 157-179.

Department of Mathematics Education

Inha University

Incheon 402-751, Korea

E-mail: qheung@inha.ac.kr

Department of Mathematics

Kunsan National University

Kunsan 573-701, Korea

E-mail: tsjung@kunsan.ac.kr 